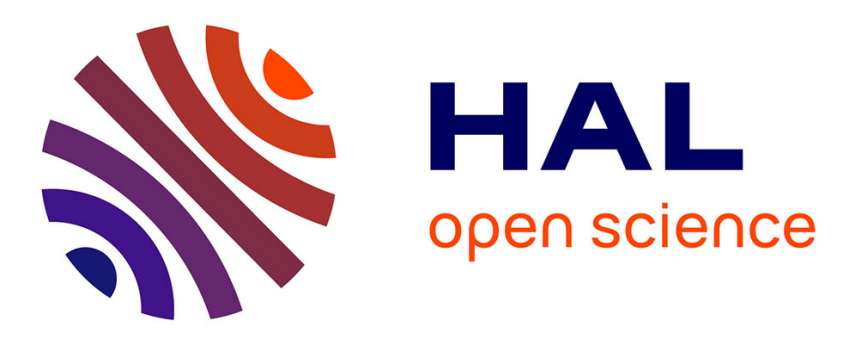

\title{
Automated On-Ramp Merging System for Congested Traffic Situations
}

Vicente Milanés, Jorge Godoy, Jorge Villagra, Joshué Pérez Rastelli

\section{To cite this version:}

Vicente Milanés, Jorge Godoy, Jorge Villagra, Joshué Pérez Rastelli. Automated On-Ramp Merging System for Congested Traffic Situations. IEEE Transactions on Intelligent Transportation Systems, 2011. hal-00737629

\author{
HAL Id: hal-00737629 \\ https://hal.inria.fr/hal-00737629
}

Submitted on 2 Oct 2012

HAL is a multi-disciplinary open access archive for the deposit and dissemination of scientific research documents, whether they are published or not. The documents may come from teaching and research institutions in France or abroad, or from public or private research centers.
L'archive ouverte pluridisciplinaire HAL, est destinée au dépôt et à la diffusion de documents scientifiques de niveau recherche, publiés ou non, émanant des établissements d'enseignement et de recherche français ou étrangers, des laboratoires publics ou privés. 


\title{
Automated On-Ramp Merging System for Congested Traffic Situations
}

\author{
Vicente Milanés, Jorge Godoy, Jorge Villagrá, and Joshué Pérez
}

\begin{abstract}
Traffic merging in urban environments is one of the main causes of traffic congestion. From the driver's point of view, the difficulty arises along the on-ramp where the merging vehicle's driver has to discern whether he should accelerate or decelerate to enter the main road. In parallel, the drivers of the vehicles already on the major road may have to modify their speeds to permit the entrance of the merging vehicle, thus affecting the traffic flow. This paper presents an approach to merging from a minor to a major road in congested traffic situations. An automated merging system that was developed with two principal goals, i.e., to permit the merging vehicle to sufficiently fluidly enter the major road to avoid congestion on the minor road and to modify the speed of the vehicles already on the main road to minimize the effect on that already congested main road, is described. A fuzzy controller is developed to act on the vehicles' longitudinal control-throttle and brake pedals-following the references set by a decision algorithm. Data from other vehicles are acquired using wireless vehicleto-infrastructure (V2I) communication. A system installed in the infrastructure that is capable of assessing road traffic conditions in real time is responsible for transmitting the data of the vehicles in the surrounding area. Three production vehicles were used in the experimental phase to validate the proposed system at the facilities of the Centro de Automática y Robótica with encouraging results.
\end{abstract}

Index Terms-Intelligent vehicles, intervehicle communication, road traffic control, road vehicle control, traffic management.

\section{INTRODUCTION}

$\mathbf{T}$ OGETHER with the considerable increase in the number of drivers over the past few decades, there has been a corresponding growth in the number of cars on the road. Of all transport areas, urban environments are the most congested. There are two main causes. On the one hand, there is road saturation in the vicinity of large cities where it is difficult to increase the number of roads because of limited construction space. On the other hand, there is the dependence on the human driver. Although advanced driver-assistance systems (ADASs) have been implemented in vehicles in recent years, the ultimate decision is the drivers' and will depend on their experience and behavior in predicting what the vehicles in their vicinity will

Manuscript received July 29, 2010; revised October 1, 2010; accepted November 27, 2010. Date of publication December 23, 2010; date of current version June 6, 2011. This work was supported by the CYCIT (Spain) and Plan Nacional (Spain) from the GUIADE (P9/08) and TRANSITO (TRA200806602-C03-01) projects, respectively. The Associate Editor for this paper was L. Li.

The authors are with the AUTOPIA program of the Center for Automation and Robotics, Universidad Politécnica de Madrid-Consejo Superior de Investigaciones Científicas, 28500 Madrid, Spain (e-mail: vicente.milanes@ car.upm-csic.es; jorge.godoy@car.upm-csic.es; jorge.villagra@car.upm-csic. es; joshue.perez@car.upm-csic.es).

Color versions of one or more of the figures in this paper are available online at http://ieeexplore.iee.org.

Digital Object Identifier 10.1109/TITS.2010.2096812 do, with the result that many of the decisions they make may be incorrect.

On-ramps constitute one of the major causes of congestion in large cities. The result may not only be long delays for the drivers but also a significant increase in pollution [1]. For the drivers, performing a ramp entrance is a complex maneuver since both the merging driver and those already on the main road have to be able to interpret the situation of the traffic around them.

Ramp entrance automation is a suitable candidate for inclusion as part of a cooperative maneuver context for congested traffic situations. There have been several advances in this area in recent years. For instance, the California Partners for Advanced Transit and Highways (PATH) program in the United States has pioneered in the development of ADAS solutions for congested traffic situations. In particular, its eight-vehicle platoon demonstration at the National Automated Highway Systems Consortium Technical Feasibility Demonstration in 1997 is one of the most impressive results involving real vehicles up to now [2].

Another approach has been to use simulations to address traffic congestion problems. Mohandas et al. [3] used an adaptive proportional integral rate controller in a single-lane traffic scenario to improve traffic congestion when traffic volume exceeds the capacity of the road. In [4], a new spacing policy is proposed for adaptive cruise control (ACC) systems in traffic jams, using the information on both the ACC vehicle's state and the braking capacity of the automated vehicle following it. A wavelet-based pattern recognition method to classify realtime vehicle velocities to estimate congestion conditions and road type is presented in [5]. A traffic simulation study of how cooperative ACC (CACC) can contribute to reducing traffic congestion is described in [6].

With respect to merging into traffic flow, the first relevant results were obtained in the 1960s. Godfrey [7] started the analysis and research of merging disciplines for automated transportation systems. After him, Brown [8] designed a control strategy to merge on automated guideway transit systems called "adaptative merging." In the 1980s, Shladover [9] studied by simulation a merge junction to increase guideway system capacity. At the beginning of 2000s, Lu et al. [10], who was working for the PATH program, developed a longitudinal control algorithm for vehicle merging based on magnetic marks installed on the infrastructure and communications.

In recent years, several simulation approaches have been described. Hidas [11] collected video-recording data on the microscopic details of merging and weaving maneuvers under congested traffic conditions and used them to develop a 


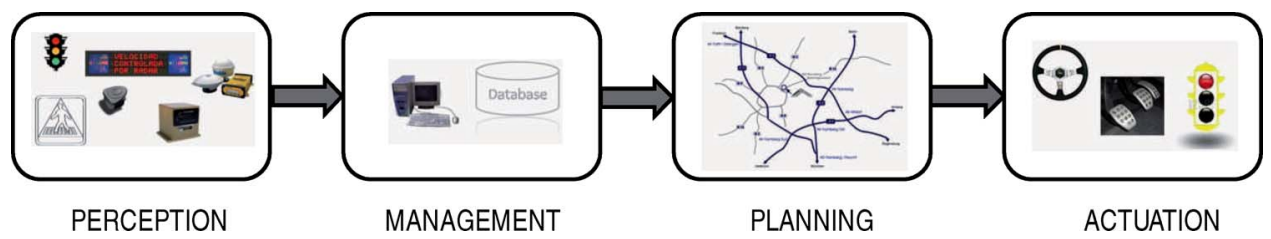

Fig. 1. AUTOPIA architecture for automatic cooperative driving.

controller that applies concepts of intelligent agents. A microscopic single-level gap-acceptance model with the observed flow allocation pattern at an on-ramp when the main road is congested is presented in [12]. Continuous kinematic wave models of merging traffic flow are studied in [13], with the proposal of a solution framework for the Riemann problem of merging traffic flow. An application to real-time toll-plaza and work-zone management using a ramp-metering algorithm is presented in [14]. The effect of heavy commercial vehicles on the capacity and overall performance of freeway ramp-merging sections is considered in [15], with an examination of the potential of deploying control strategies for freeway merging points to mitigate traffic congestion. Melo et al. [16] used an intelligent vision-based system to analyze traffic flow in onramp scenarios, with the goal being to include it in driving aid systems.

While the results of these simulations are encouraging, their translation to the real world is far from straightforward since gasoline-propelled vehicle dynamics at very low speeds-as is the case in congested traffic situations-are highly nonlinear and difficult to adequately model. This paper describes a proposal to automate on-ramp entrances to main roads to improve traffic congestion using real vehicles. To that end, a decision algorithm computes a target reference distance for each vehicle to ensure a smooth transition between a predefined merging zone on the on-ramp and the final merging point. With the assumption that the drivers are capable of maintaining the vehicles in their lane, a fuzzy longitudinal controller was developed to permit the vehicle approaching from the minor road to merge into the main road by tracking as precisely as possible the intervehicle distance references provided by the decision algorithm. For this purpose, a local control station (LCS) is responsible for detecting the following: 1) when the vehicle is to merge into the main road traffic and 2) between which two vehicles is the best option. Three vehicles are therefore involved in the maneuver, i.e., the merging vehicle and the leading and trailing vehicles between which it will have to merge. Both the trailing and the merging vehicles have to be controlled so that the maneuver affects the main road traffic as little as possible.

In brief, the main contributions in this paper are given as follows:

1) vehicle-to-infrastructure (V2I)-based intelligent control algorithm to compute in advance the optimal distance to perform the merging affecting the major road as little as possible;

2) design and development of a valid controller based on fuzzy logic for merging in congested traffic situations;

3) implementation of the whole system in gas-propelled production cars testing the system at very low speeds, where vehicle dynamics are highly nonlinear.
The rest of this paper is structured as follows: Section II presents the AUTOPIA program's control architecture that is designed to deal with cooperative automated vehicle maneuvers. The real vehicles involved in the maneuver and the LCS are described in Section III. The proposed solution, including both the decision and the control algorithm, is explained in Section IV. Section V presents the experimental results obtained on the Center for Automation and Robotics (CAR) driving circuit. Finally, Section VI gives the conclusions drawn from the work.

\section{Control Architecture}

This work is part of the AUTOPIA program, whose longterm goal is the development of automatic cars using massproduced vehicles and tests on real roads [17]. Previous works [18], [19] have presented the AUTOPIA architecture designed for an automatic car, which is a classical perception-planningactuation scheme. Our present goal is to move on to the traffic control of cooperating vehicles [20]. Roughly speaking, this involves an LCS [21], which receives information from the vehicles in its domain, analyzes this information to determine when a potentially risky situation might arise, and notifies the vehicles of maneuvers they have to do. To this end, a modification was made to our control architecture by introducing a new stage: management. Fig. 1 shows the stages.

1) Perception: In this stage, sensor information is sent from the vehicles (cars, trucks, motorbikes, etc.) and the infrastructure (traffic signals, light panels, etc.) to the LCS. For the vehicles, this information may come from a Global Navigation Satellite System, an Inertial Measurement Unit, a compass, or some other sensor unit (e.g., a controller area network (CAN) bus). For the infrastructure, there might also be sensors installed (e.g., Zigbee [22] or radio-frequency identification [23] systems) to provide extra information. The LCS is limited by the area that can be covered by the local communication system. Such infrastructure limitations are not taken into account in this paper.

2) Management: The LCS analyzes and organizes the sensor information it receives to find the best way to resolve risky traffic situations by taking into account the state of the traffic and the vehicles driving in the area. The LCS will then transmit the relevant information as data to the vehicles and the infrastructure to permit them to make safe maneuvers.

3) Planning: With the information transmitted from the LCS, the vehicles and the infrastructure evaluate the conditions and choose the best alternative that will improve traffic flow. 


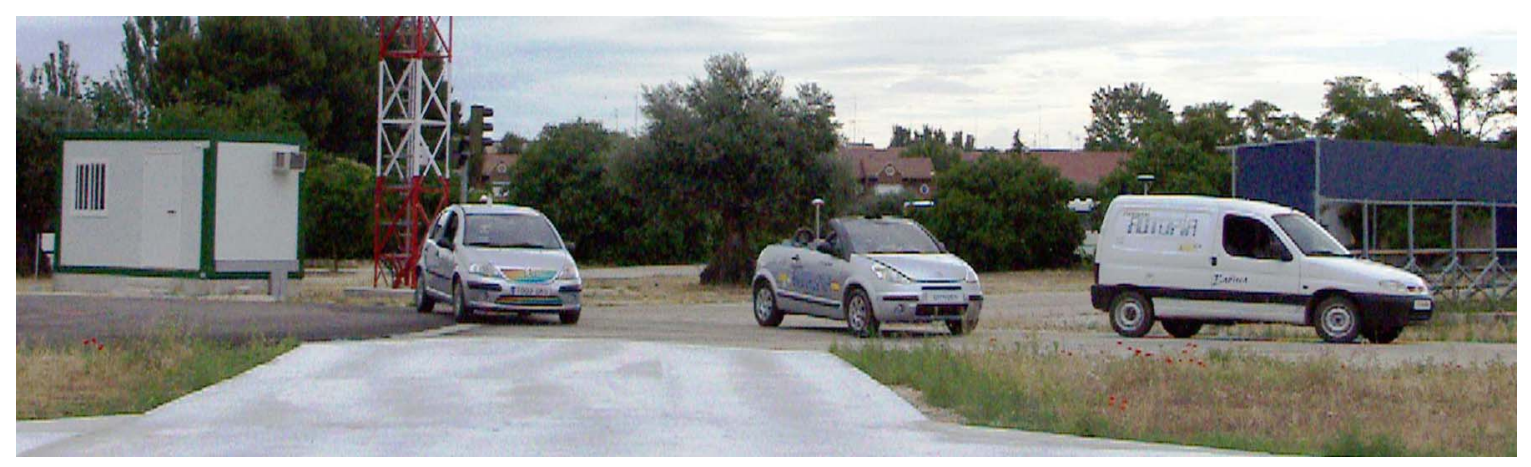

Fig. 2. Prototype vehicles and LCS for the experimental phase.

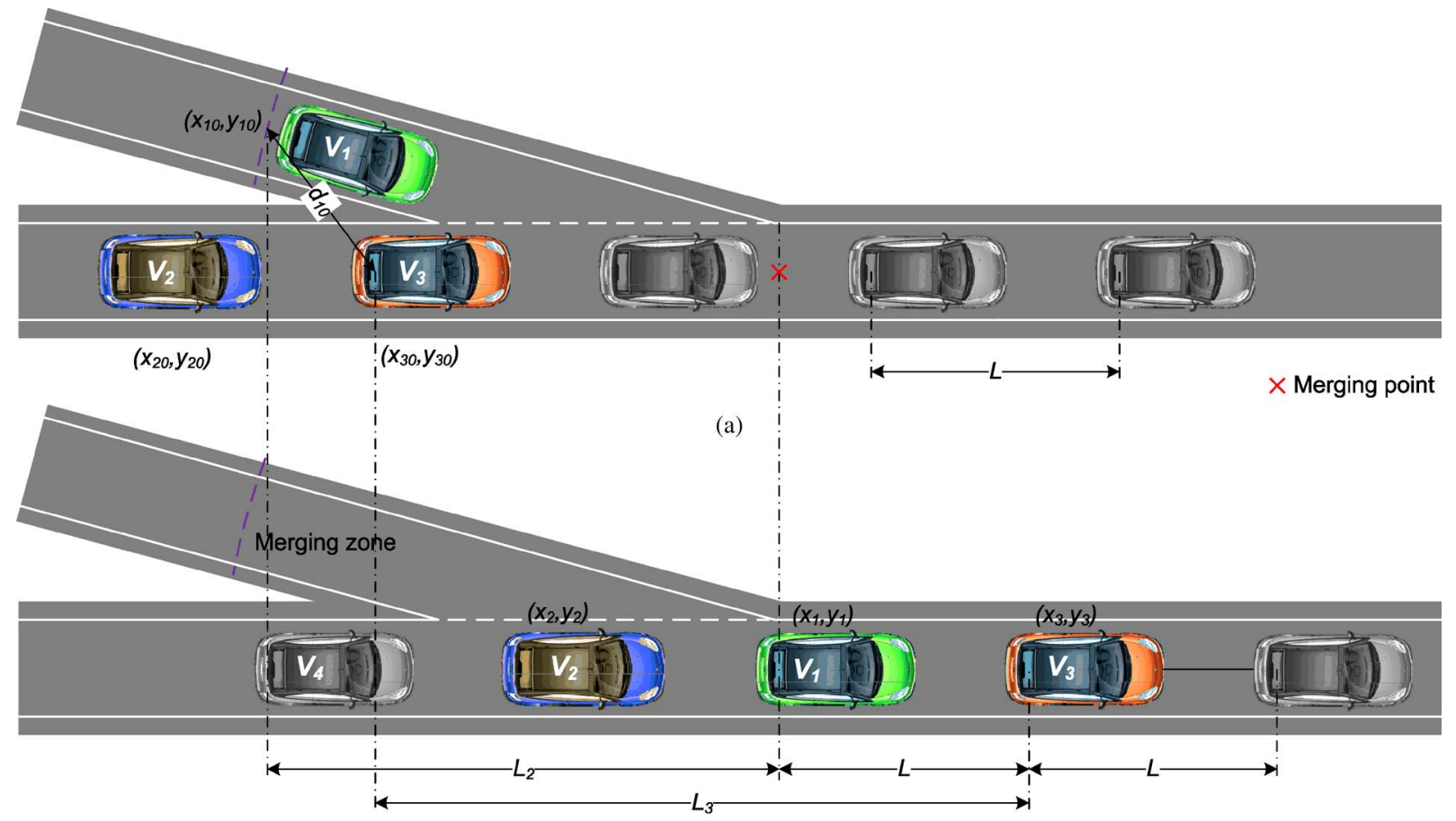

(b)

Fig. 3. Scheme of the ramp entrance situation. (a) Merging vehicle enters the control station's area of influence. (b) Vehicles' positions after the merging maneuver.

4) Actuation: In the last stage, the options selected in the previous stage are sent to the vehicles' actuators. For example, in the case of a traffic light, this may be to change the light; in the case of a vehicle, it may be to modify its speed.

\section{PRototy Pe Vehicles AND LOCAL CONTROL STATION}

This section describes the mass-produced vehicles and the LCS used to test the automatic ramp entrance system (see Fig. 2). It will describe the automation process of three vehicles, i.e., two gasoline-propelled and one electric vehicle. Then, the installation and the operation of the LCS will be presented.

\section{A. Gasoline-Propelled Automated Vehicles}

Two Citroën C3s, one being a convertible, are used. Since their mechanical characteristics are similar, the same systems were installed in both. To implement an automatic ramp en- trance system, only longitudinal control is needed. Both vehicles have an automatic gearbox; therefore, the automation process is limited to throttle and brake pedals.

For the throttle, an analog card was installed in the onboard control unit (OCU) of each car, generating a signal that represents the pressure on the pedal. For the speed control, the control loop is closed using CAN bus messages.

The brake is controlled using an electrohydraulic braking system consisting of three valves to manage the pressure that is applied to the wheels. This system is controlled via a CAN card connected to the OCU using a CAN/USB converter [24].

\section{B. Electric Automated Vehicle}

The electric vehicle used in the experimental phase is a Berlingo Citroën van. This vehicle is the leading vehicle in the picture of the ramp entrance in Fig. 3. This vehicle therefore only requires speed control.

Since this vehicle is not equipped with a CAN bus, a differential Hall effect sensor was installed. This sensor was coupled 
to a cogwheel attached to one of the front wheels of the car. The output of the sensor is connected to the OCU using an analog-to-digital converter. Speed control was implemented by transmitting the analog value corresponding to the desired level of pressure on the throttle pedal via an analog CAN card.

\section{C. $L C S$}

An LCS is responsible for detecting risky traffic situations in its control area and then advising the vehicles involved.

A wireless access point is used to detect any vehicle that is in its vicinity, establishing a V2I communication. An industrial personal computer is programmed to analyze the information proceeding from the vehicle (position, speed, and intention) or the infrastructure to inform the vehicle about the traffic situation in which it is driving. With this information, the vehicles are capable of selecting among various fuzzy controllers the appropriate action to impose on their actuators.

\section{Automated Ramp Entrance System}

The automatic system to carry out the control has to be a compromise between the theoretically optimal solution of the merging vehicle's joining the traffic on the main road and the need to avoid any harsh braking on the part of the trailing vehicle, which could lead to a chain of simultaneous braking on the part of the rest of the cars on the major road.

The design of the system was divided into three phases:

1) detecting the vehicle driving along the minor road that will merge into the main road traffic. The LCS is responsible for this action. It detects when a vehicle is driving along the on-ramp and selects which cars on the major road will be the leading and trailing pair in the merge. To this end, the position and speeds of each car involved in the maneuver have to be sent to the LCS for it to determine the best option;

2) developing an algorithm that is capable of optimizing the merge to affect the traffic on the main road as little as possible. As a result of this algorithm, the variables required to perform the automated longitudinal control will be sent as reference data to the merging and trailing vehicles for them to follow;

3) designing an intelligent controller to follow the reference data generated by the preceding algorithm.

\section{A. Decision System}

The merging control system has to ensure that sufficient headway is achieved by the time the merging point is reached in order for the merge to be smooth without collision. To that end, a virtual vehicle [25] is introduced by mapping the merging vehicle onto the object lane in the main road.

Assume that the spacing between all the vehicles on the main road is controlled to be $L$. The relationship established between the merging vehicle $V_{1}$ and the leading vehicle $V_{3}$ will have to guarantee a spacing of $L$ at the merging point. For the trailing vehicle $V_{2}$, the longitudinal control will have to target a $2 L$ spacing, with the leading vehicle at the merging point.
To perform this task, the reference (target) distances of each vehicle will be computed as follows:

Once the decision system has been enabled, the reference distances $d_{r_{1}}$, i.e., the desired $V_{1}$-to- $V_{3}$ distance, and $d_{r_{2}}$, i.e., the desired $V_{2}$-to- $V_{3}$ distance, that will be transmitted to the merging and trailing vehicles can be written as

$$
\begin{aligned}
& d_{r_{1}}=d_{1_{0}}+s_{1} \\
& d_{r_{2}}=L+s_{2}
\end{aligned}
$$

where $d_{1_{0}}$ is the initial distance between vehicles $V_{1}$ and $V_{3}$, and $s_{1}$ and $s_{2}$ are the reference distance variations of the merging and trailing vehicles, respectively. Variables $s_{1} \in\left[0, L-d_{1_{0}}\right]$, $s_{2} \in[0, L]$ are defined to allow the reference distances for the merging and trailing vehicles to be $L$ and $2 L$, respectively, at the end of the merging maneuver. These normalized distances $s_{1}$ and $s_{2}$ can be expressed in terms of the real positions of the merging and leading vehicles $x_{1}$ and $x_{3}$, respectively, i.e.,

$$
\begin{aligned}
& s_{1}(t)=\frac{\left(L-d_{1_{0}}\right)\left(x_{3}(t)-x_{3_{0}}\right)}{L_{3}} \\
& s_{2}(t)=\frac{\left(x_{1}(t)-x_{1_{0}}\right) L}{L_{1}} .
\end{aligned}
$$

Note that only $x_{1}$ and $x_{3}$ are time-dependent variables. The rest of the parameters are geometric constants; $L_{1}$ and $L_{3}$ are the distances shown in Fig. 3, and $\left(x_{1_{0}}, y_{1_{0}}\right)$ and $\left(x_{3_{0}}, y_{3_{0}}\right)$ are the initial positions of the merging and leading vehicles, respectively. In addition, $d_{r_{2}}$ depends only on $x_{1}(t)$ - and is independent of $y_{1}(t)$-because the trailing vehicle $\left(V_{2}\right)$ takes as reference the virtual vehicle of $V_{1}$, which is mapped onto the platoon lane.

The final reference distances are therefore

$$
\begin{aligned}
& d_{r_{1}}=\left\{\begin{array}{cl}
d_{1_{0}}+\frac{\left(L-d_{1_{0}}\right)\left(x_{3}-x_{3_{0}}\right)}{L_{3},} & \text { if }\left(x_{3}-x_{3_{0}}\right)<L_{3} \\
L_{3} & \text { if }\left(x_{3}-x_{3_{0}}\right) \geqslant L_{3}
\end{array}\right. \\
& d_{r_{2}}=\left\{\begin{array}{cl}
L+\frac{\left(x_{1}-x_{1_{0}}\right) L}{L_{1},}, & \text { if }\left(x_{1}-x_{1_{0}}\right)<L_{1} \\
2 L & \text { if }\left(x_{1}-x_{1_{0}}\right) \geqslant L_{1} .
\end{array}\right.
\end{aligned}
$$

Remark 1: Instead of a linear function between the reference distance and the vehicle displacement, a higher order polynomial could have been used to smooth the transition from the starting to the merging point. However, in several experimental trials, the control system was found to manage the linear solution better than the a priori smoother strategy. This result can be explained by the fact that, with the smoother reference trajectory at the start and end points, the control action has to more rapidly change to meet the target spacing at the merging point; therefore, the tracking of the reference distance worsens.

The preceding decision system was implemented on a simulator, placing three kinodynamic vehicle models [26] in a merging situation. The same longitudinal controller was used in each vehicle to track the desired spacing.

To illustrate the algorithm's ability to handle different entrance positions of the platoon, two different simulations are shown in Figs. 4 and 5. In the first scenario, when the merging vehicle triggers the control algorithm located in the 

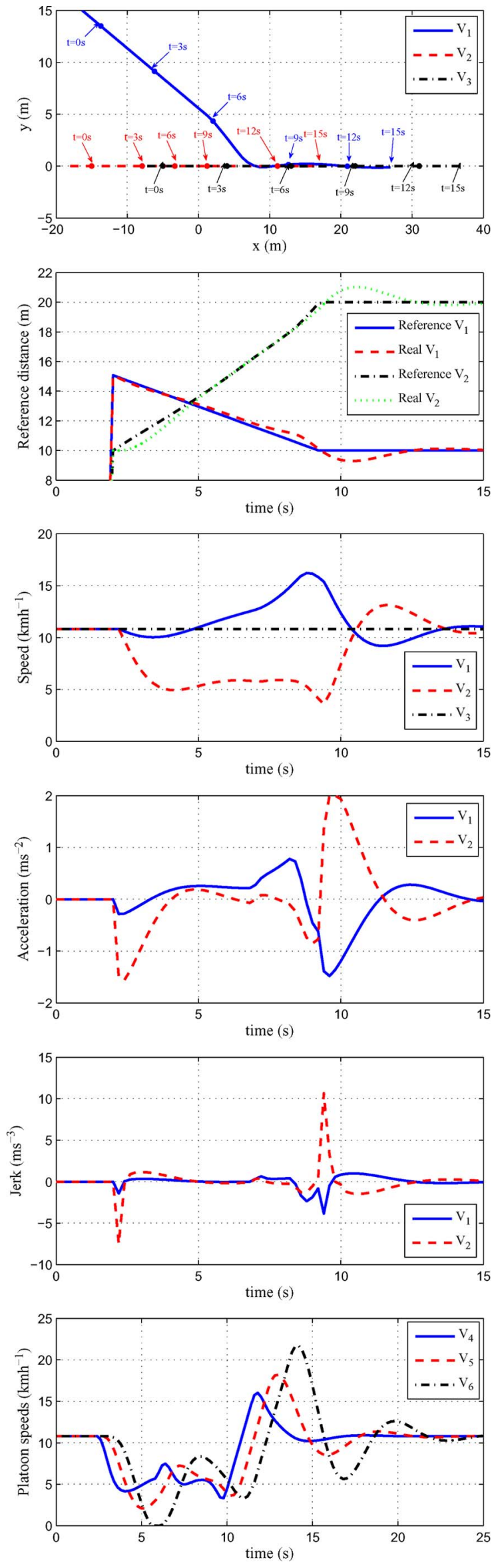

Fig. 4. Evolution of the vehicles in the first simulation scenario.
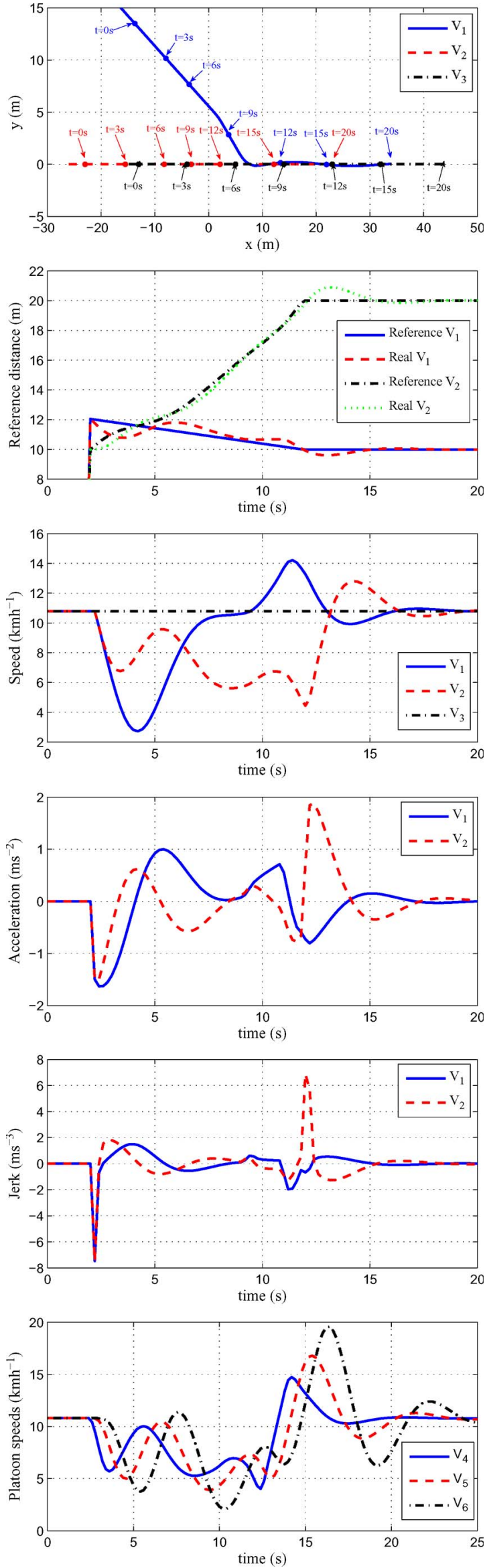

Fig. 5. Evolution of the vehicles in the second simulation scenario. 
control station, vehicles 2 and 3 are located at $x_{2_{0}}=-18$ and $x_{3_{0}}=-8$, respectively. In the second scenario, the platoon vehicles (2 and 3) are farther from the merging point than in the first case $\left(x_{2_{0}}=-26, x_{2_{0}}=-16\right)$. In both scenarios, the sampling rate is constrained by the Global Positioning System (GPS) frequency ( $T_{s}=0.2 \mathrm{~s}$ ), the spacing $L$ is equal to $10 \mathrm{~m}$, and the initial speed for all three vehicles is $3 \mathrm{~ms}^{-1}$, which is a speed that vehicle 3 maintains constant throughout the maneuver.

Note that the reference distance of vehicle 2 smoothly evolves from $L$ to $2 L$ in both cases, but the time interval for the transition to take place differs between the two cases. While, due to the different starting positions of the two scenarios, the reference distances are only slightly different for both vehicles 1 and 2, the evolution of their speeds is notably different. This is also reflected in the acceleration and jerk although their values are reasonable in both simulations.

The bottom plot in Figs. 4 and 5 shows the evolution of the traffic behind vehicle $V_{2}$. Three vehicles, i.e., $V_{4}, V_{5}$, and $V_{6}$, were added in the major road. One can see that the smaller the distance from $V_{2}$ to the merging point, the greater the effect on the downstream traffic. The results indicate that an optimal determination of the interdistance among vehicles on the major road, which is typically set at $2 \mathrm{~s}$ in production cars [27], would improve the traffic situation.

This decision algorithm is capable of determining the appropriate reference distance independently of the angle between the major and the minor roads; $d_{1_{0}}$ is diagonally measured to eliminate the dependence on the angle of the merging lane. With the assumption that the drivers are capable of maintaining the vehicles in their lane, the reference distance will be as smooth as possible, taking into account the actual distance from the merging vehicle to the merging point. Thus, the decision algorithm is applicable to any road layout.

\section{B. Control System}

Fuzzy logic is a powerful problem-solving methodological approach with a wide range of applications to real-world problems. Whereas classical controllers require exact equations and precise numerical values, fuzzy controllers provide solutions based on vague, ambiguous, or imprecise information about the system to be controlled. In the automotive sector, it is used in several processes because of its simplicity and flexibility in handling problems with imprecise and incomplete data. An example is the automatic transmission system on the Volkswagen Jetta, which models driver behavior.

The choice of fuzzy logic as control technique is based on both the AUTOPIA program experience in this soft computing technique and the results obtained in [28], where four advanced control techniques in vehicular applications are compared with excellent results for the fuzzy system. Furthermore, it can incorporate human procedural knowledge into control algorithms, and it allows the designer to partially mimic human driving behavior. Fuzzy logic starts with and builds on a set of usersupplied human language rules. To this end, a fuzzy inferenceengine-denominated Ordenador Borroso EXperimental had previously been developed at CAR. It uses Mamdani inference,
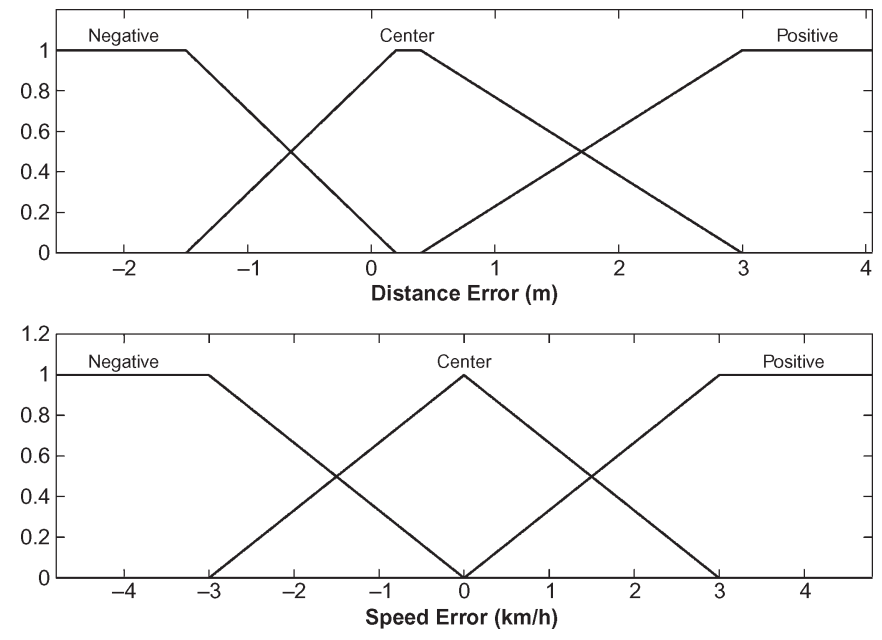

Fig. 6. Definition of the membership functions for the input variables.

with singleton-type membership functions to codify the output variables.

Two input variables were used for the control: 1) SpeedError, which is defined as the difference between the leading and the trailing vehicles' speeds (in kilometers per hour), and 2) DistanceError, which is defined as the difference between the desired and actual gap between the cars (in meters). The membership function definitions for each input variable are shown in Fig. 6.

The fuzzy input variable SpeedError contains three membership functions for each of its three associated linguistic labels. The Center linguistic label is used to indicate that the trailing vehicle is following the leading vehicle at the same speed. The margin or error is set at $\pm 3 \mathrm{~km} / \mathrm{h}$. The Positive linguistic label is used to accelerate the car. Analogously, the Negative linguistic label is used to brake the car.

The second fuzzy input variable is DistanceError. This fuzzy input also has three membership functions defined for three associated linguistic labels. Note that these fuzzy membership functions are defined as asymmetric. The Center linguistic label is used to indicate that the trailing vehicle is maintaining the desired gap with respect to the leading car. This membership function is trapezoidal. The reason is that the differential GPS (DGPS) errors in the vehicles' positioning have to be allowed for, with distance errors of less than $\pm 20 \mathrm{~cm}$ being acceptable. The Negative and Positive linguistic labels (to accelerate or brake the car, respectively) have different slopes. This is because the automated braking system [24] was designed for risk situations that demand strong action on the brake pedal, but a gentler action was considered for the present application.

The output of the controller is the action on the throttle and brake pedals defined as Sugeno singletons (see Fig. 7). Thus, the output variable Pedal determines which actuator has to be pressed and the magnitude of the action. The possible output values are within the range $[-1,1]$, where -1 indicates that the brake pedal is completely depressed, and 1 indicates that maximum action is applied to the accelerator pedal. 


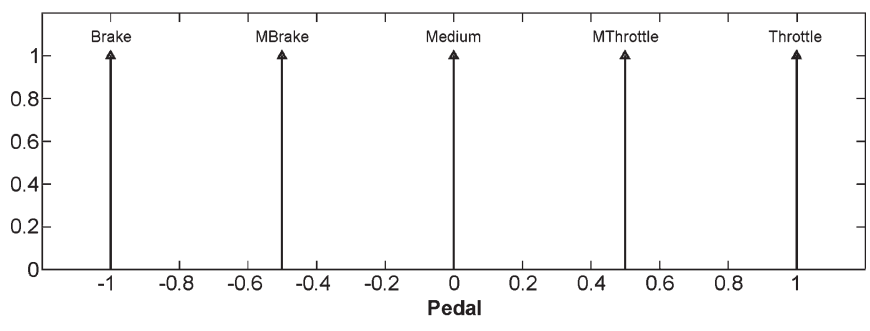

Fig. 7. Definition of the membership functions for the output variables.

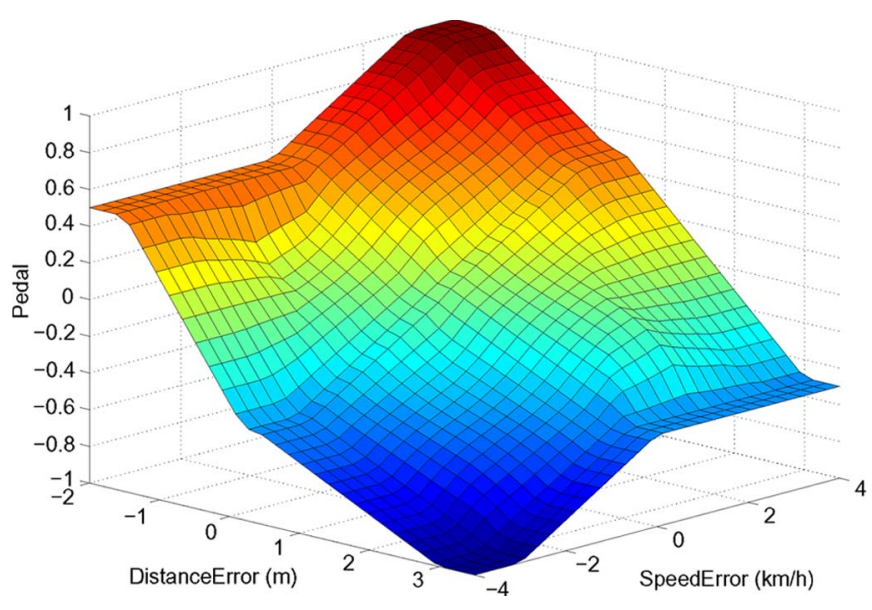

FIg. 8. Control surface for the fuzzy control system.

Fig. 8 shows the control surface for the output variable Pedal as a function of the fuzzy input variables SpeedError and DistanceError. The smoothness in the variation of the surface is indicative of the suitability of the rules selected. Given the low speeds considered in this application because of the limitations of CAR's driving circuit, the action on the throttle was weighted at $40 \%$, and that on the brake was weighted at $10 \%$.

\section{EXPERIMENTS}

The proposed algorithm was tested on the experimental driving circuit at CAR's facilities. This circuit represents an inner city area with a combination of straight-road segments, curves, roundabouts, incorporations, and crossroads. In the tests, the three vehicles described in Section II were used.

The trials consisted of two vehicles driving along the main road emulating a congested traffic situation and performing ACC between them at low speed. The leading vehicle was the electric van, which was named Rocinante, and the trailing vehicle was the Citroën $\mathrm{C} 3$ named Platero. At the same time, the convertible Citroën $\mathrm{C} 3$, which was called Clavileño, was driven along the minor road to merge into the main road. We assume that the main road is congested and that the control algorithm via the LCS has been capable of distinguishing the two target cars of the main road platoon between which to merge to minimize the effect on the main road traffic. As, experimentally, we only have three vehicles available to carry out these trials, the LCS indicates to
Clavileño that the best option to merge is between Rocinante and Platero.

Fig. 9 shows the results of the system during one experiment. The top graph plots the evolution of the positions of the vehicles as one of them is detected to be merging into the main road, taking the merging point as the origin of coordinates $(0,0)$. The three trajectories were obtained via each car's DGPS. The middle graph plots the vehicles' speeds during the experiment. Time 0 represents the moment at which the automated merging system is activated. The bottom graph represents the distance between the vehicles. This value was set at $12 \mathrm{~m}$ as the reference spacing when the merging vehicle reaches the merging point.

At the beginning, the merging vehicle's speed roughly follows its predefined target up to time 0 , which is the instant at which the automated merging system is activated. Since the main road vehicles' distances to the merging vehicle are still large, the speed of each car is maintained up to time $7 \mathrm{~s}$. One then can see how the trailing vehicle reduces its speed to permit the merging vehicle to enter the main road. Consequently, the distance between the trailing and leading vehicles increases. The speeds of the trailing and merging vehicles are modified to achieve the reference spacing at the merging point from time $7 \mathrm{~s}$ to time $20 \mathrm{~s}$. During this interval, the trailing vehicle's speed is adapted without stopping the car, affecting the traffic as little as possible. The merging vehicle reaches the merging point at time $20 \mathrm{~s}$. As can be seen, the values of the spacing between the three vehicles-between Rocinante and Clavileño and between Clavileño and Platero-are close to $12 \mathrm{~m}$. From that instant onward, the three vehicles perform an ACC. A video file showing the automated maneuver at CAR's facilities can be retrieved from http://www.iai.csic.es/autopia/Videos/Merging.wmv.

\section{CONCLUSION}

Improving traffic flow is one of the priority objectives of transportation authorities. This paper has presented an approach to solving one of the major causes of congestion in urban environments: merging from minor to major roads. To this end, a control algorithm has been developed to decide when the merging vehicle has to enter the main road, and a fuzzy controller has been designed to manage the vehicle's actuator to follow the reference set by the control algorithm. This solution has been validated first in simulations and then in real experiments. Three vehicles have been used in the experimental phase, with two having automated throttle and brake. Finally, a V2I-based communication system has been used to permit the vehicles to exchange information.

The real experiments consisted of several trials conducted at low speeds. The results have been promising, showing that the system is capable of predicting the best option for the merging vehicle to enter the main road. The speeds of the vehicles involved in the maneuver have been automatically modified in advance to carry out the merge as smoothly as possible. Taking into account the merging effects over the major road in simulation, the next steps in our AUTOPIA research program will focus on introducing more cars to study the effects on the main road's traffic flow. 


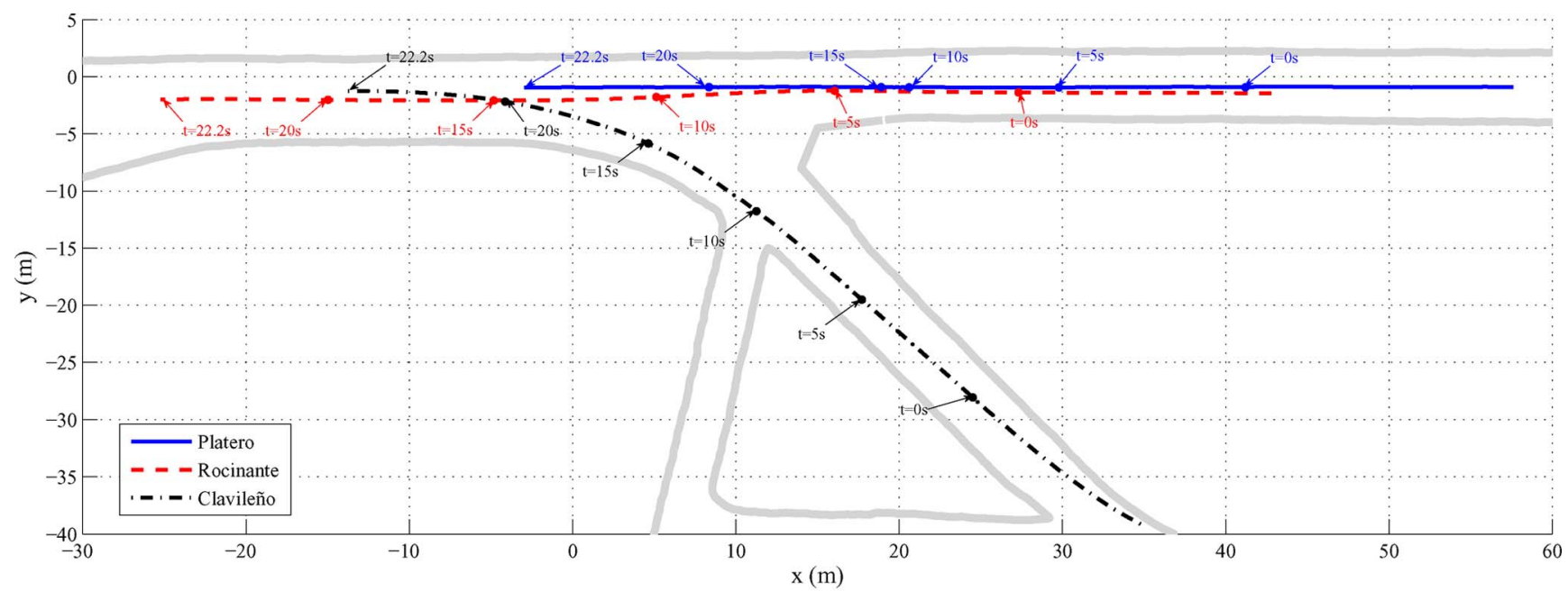

(a)

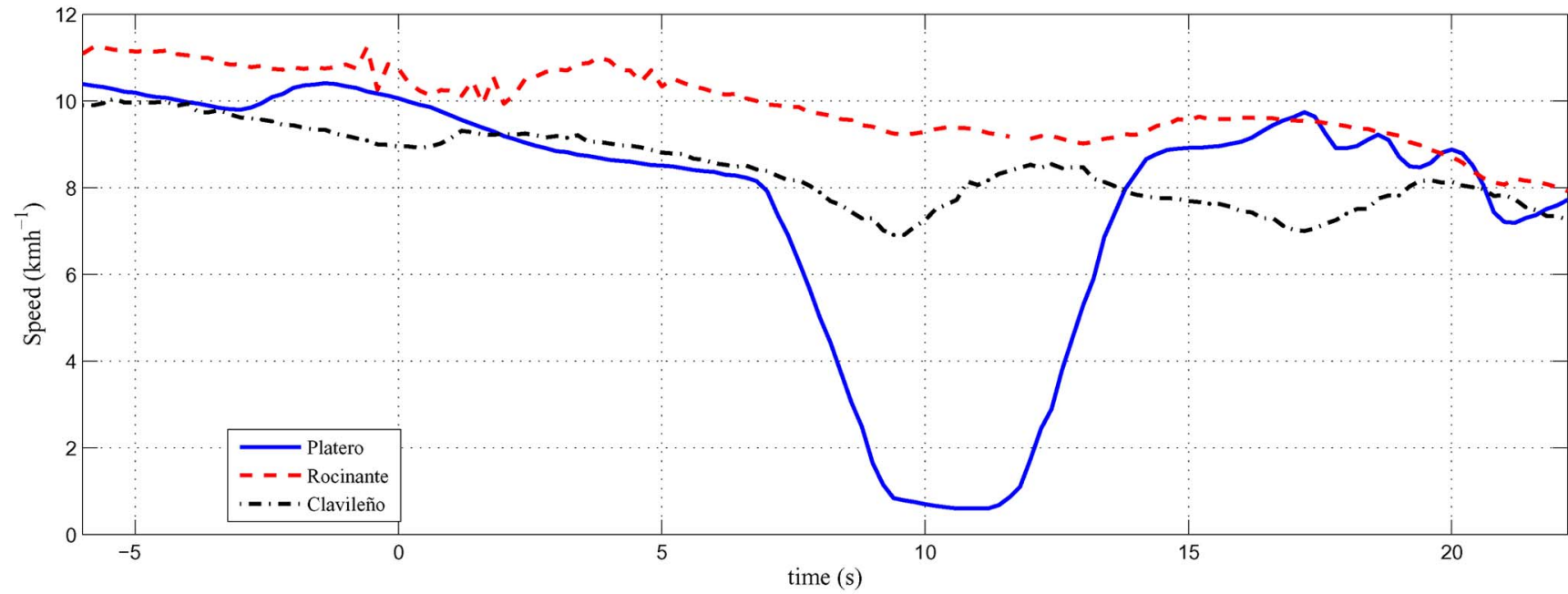

(b)

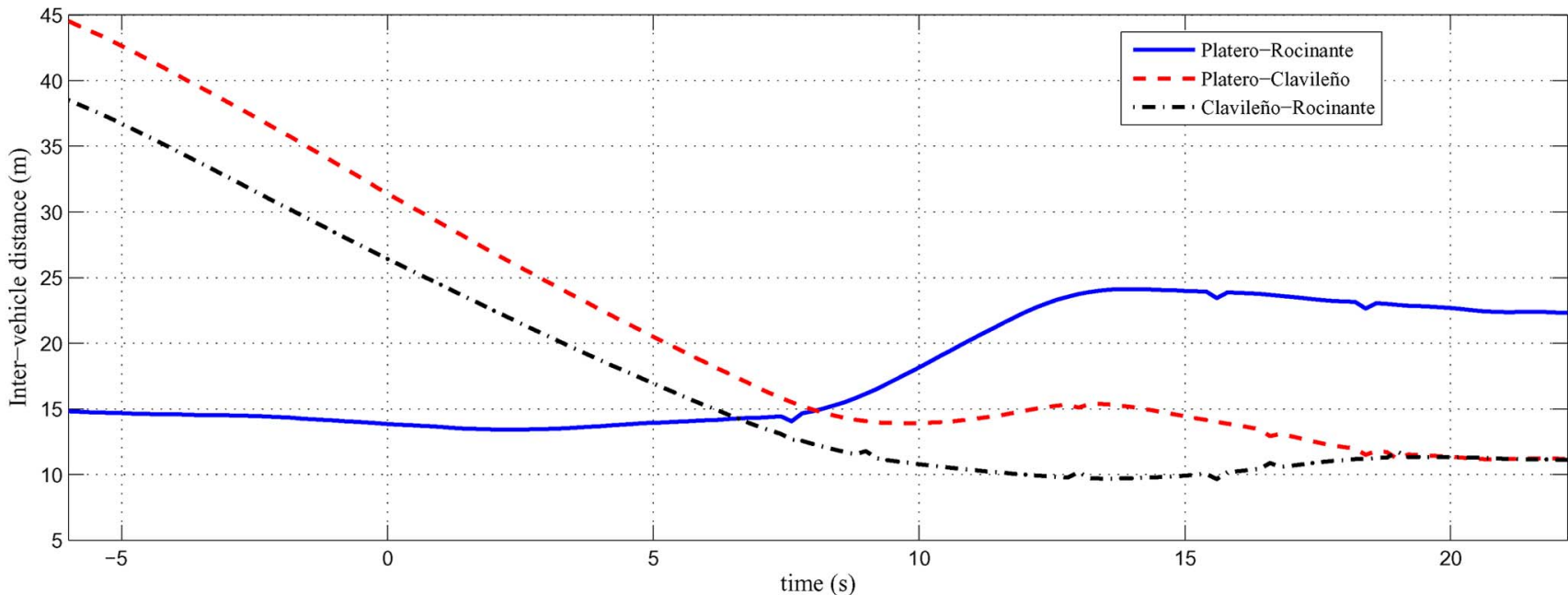

(c)

Fig. 9. Evolution of three vehicles in a real experiment. (a) $X-Y$ position. (b) Speeds. (c) Relative distances between vehicles.

\section{REFERENCES}

[1] G. Bel and M. Nadal, "Anuario de la movilidad 2008,” Real Automóvil Club de Cataluña, Barcelona, Spain, Tech. Rep., 2009.

[2] R. Rajamani, H. Tan, B. Law, and W. Zhang, "Demonstration of integrated longitudinal and lateral control for the operation of automated vehicles in platoons," IEEE Trans. Control Syst. Technol., vol. 8, no. 4, pp. 695-708, Jul. 2000.
[3] B. K. Mohandas, R. Liscano, and O. Yang, "Vehicle traffic congestion management in vehicular ad-hoc networks," in Proc. IEEE 34th Conf. $L C N, 2009$, pp. 655-660.

[4] J. Zhao, M. Oya, and A. El Kamel, "A safety spacing policy and its impact on highway traffic flow," in Proc. IEEE Intell. Vehicles Symp., 2009, pp. 960-965. 
[5] W. Zhu and M. Barth, "Vehicle trajectory-based road type and congestion recognition using wavelet analysis," in Proc. IEEE ITSC, 2006, pp. 879-884.

[6] B. van Arem, C. J. G. van Driel, and R. Visser, "The impact of cooperative adaptive cruise control on traffic-flow characteristics," IEEE Trans. Intell. Transp. Syst., vol. 7, no. 4, pp. 429-436, Dec. 2006.

[7] M. B. Godfrey, "Merging in automated transportation system," Ph.D. dissertation, Mass. Inst. Technol., Cambridge, MA, 1968.

[8] S. Brown, "Adaptive merging under car-follower control," Johns Hopkins Univ., Baltimore, MD, Tech. Rep., 1974.

[9] S. E. Shladover, "Operation of merge junctions in a dynamically entrained automated guideway transit system," Transp. Res. Part A: Gen., vol. 14, no. 2, pp. 85-112, Apr. 1980.

[10] X.-Y. Lu, H.-S. Tan, S. E. Shladover, and J. Hedrick, "Implementation of longitudinal control algorithm for vehicle merging," in Proc. 5th Int. Symp. Adv. Vehicle Control, Ann Arbor, MI, Aug. 2000, pp. 25-32.

[11] P. Hidas, "Modelling vehicle interactions in microscopic simulation of merging and weaving," Transp. Res. Part C, vol. 13, no. 1, pp. 37-62, Feb. 2005.

[12] E. Chevallier and L. Leclercq, "Do microscopic merging models reproduce the observed priority sharing ratio in congestion?" Transp. Res. Part C, vol. 17, no. 3, pp. 328-336, Jun. 2009.

[13] W. Jin, "Continuous kinematic wave models of merging traffic flow," Transp. Res. Part B, vol. 44, no. 8/9, pp. 1084-1103, Sep.-Nov. 2010.

[14] M. Papageorgiou, I. Papamichail, A. Spiliopoulou, and A. Lentzakis, "Real-time merging traffic control with applications to toll plaza and work zone management," Transp. Res. Part C, vol. 16, no. 5, pp. 535-553, Oct. 2008.

[15] M. Sarvi and M. Kuwahara, "Using its to improve the capacity of freeway merging sections by transferring freight vehicles," IEEE Trans. Intell. Transp. Syst., vol. 9, no. 4, pp. 580-588, Dec. 2008.

[16] J. Melo, A. Naftel, A. Bernardino, and J. Santos-Victor, "Detection and classification of highway lanes using vehicle motion trajectories," IEEE Trans. Intell. Transp. Syst., vol. 7, no. 2, pp. 188-200, Jun. 2006.

[17] [Online]. Available: http://www.iai.csic.es/users/autopia/

[18] J. Perez, C. Gonzalez, V. Milanes, E. Onieva, J. Godoy, and T. de Pedro, "Modularity, adaptability and evolution in the AUTOPIA architecture for control of autonomous vehicles," in Proc. IEEE ICM, Apr. 14-17, 2009, pp. 1-5.

[19] V. Milanés, J. Alonso, C. González, L. Bouraoui, J. Ploeg, and T. de Pedro, "Cooperative maneuvering in close enviroments among cycabs and dual-mode cars," IEEE Trans. Intell. Transp. Syst., under review. DOI: 10.1109/TITS.2010.2050060.

[20] V. Milanés, J. Pérez, E. Onieva, and C. González, "Controller for urban intersections based on wireless communications and fuzzy logic," IEEE Trans. Intell. Transp. Syst., vol. 11, no. 1, pp. 243-248, Mar. 2010.

[21] V. Milanés, J. Godoy, J. Pérez, B. Vinagre, C. González, E. Onieva, and J. Alonso, "V2i-based architecture for information exchange among vehicles," in Proc. 7th Symp. Intell. Auton. Vehicles, 2010.

[22] V. Milanés, E. Onieva, B. Vinagre, C. González, J. Pérez, and J. Alonso, "Sistema de asistencia a la conducción basado en una red de comunicaciones de bajo coste," DYNA, vol. 85, no. 3, pp. 245-254, Apr. 2010.

[23] J. Pérez, F. Seco, V. Milanés, A. Jiménez, J. Díaz, and T. de Pedro, "An RFID-based intelligent vehicle speed controller using active traffic signals," Sensors, vol. 10, no. 6, pp. 5872-5887, 2010.

[24] V. Milanés, C. González, J. E. Naranjo, E. Onieva, and T. de Pedro, "Electro-hydraulic braking system for autonomous vehicles," Int. J. Automot. Technol., vol. 11, no. 1, pp. 89-95, 2010.

[25] T. Sakaguchi, A. Uno, and S. Tsugawa, "Inter-vehicle communications for merging control," in Proc. IEEE IVEC, 1999, vol. 1, pp. 365-370.

[26] J. Villagra, D. Herrero-Perez, and M. Abderrahim, "Robust flatness-based control of an agv under varying load and friction conditions," in Proc. IEEE ICCA, 2009, pp. 1621-1628.

[27] J. E. Naranjo, C. González, R. García, and T. de Pedro, "Acc +stop\&go maneuvers with throttle and brake fuzzy control," IEEE Trans. Intell. Transp. Syst., vol. 7, no. 2, pp. 213-225, Jun. 2006.

[28] V. Milanés, J. Villagrá, J. Pérez, and C. González, "Low-speed longitudinal controllers for mass-produced cars: A comparative study," IEEE Trans. Ind. Electron., 2010, submitted for publication.

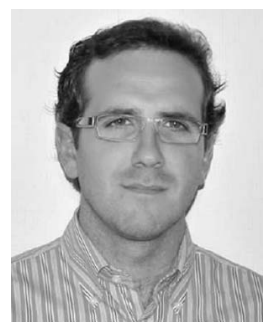

Vicente Milanés was born in Badajoz, Spain, in 1980. He received the B.E. and M.E. degrees in electronic engineering from Extremadura University, Badajoz, Spain, in 2002 and 2006, respectively, and the $\mathrm{Ph} . \mathrm{D}$. degree in electronic engineering from Alcala University, Madrid, Spain, in 2010.

Since 2006, he has been a member of the Spanish National Research Council with the Center for Automation and Robotics, Universidad Politécnica de Madrid-Consejo Superior de Investigaciones Científicas, Madrid. His research interests include autonomous vehicles, fuzzy-logic control, intelligent traffic and transport infrastructures, vehicle-infrastructure cooperation, and intelligent transportation systems.

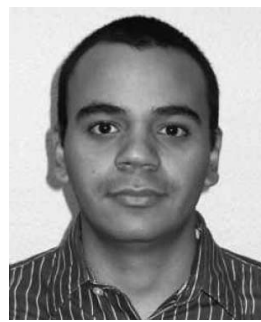

Jorge Godoy was born in Maracay, Venezuela, in 1986. He received the M.E. degree in electronic engineering from the Universidad Simón Bolívar, Caracas, Venezuela, in 2008. He is currently working toward the $\mathrm{Ph} . \mathrm{D}$. degree in automation and robotics with the Universidad Politécnica de Madrid, Madrid, Spain.

Since 2009, he has been with the Center for Automation and Robotics, Universidad Politécnica de Madrid-Consejo Superior de Investigaciones Científicas, Madrid, researching autonomous vehicles. His research interests include fuzzy-logic control and intelligent transportation systems.

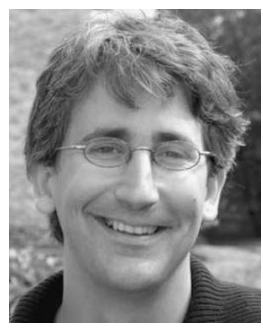

Jorge Villagrá received the M.E. degree in electrical engineering from the Universidad Politécnica of Madrid, Madrid, Spain, in 2002 and the Ph.D. degree in real-time computer science, robotics, and automatic control from the Ecole des Mines de Paris, Paris, France, in 2006.

From 2006 to 2009, he was a Research Assistant with INRIA-Rocquencourt, Le Chesnay, France, and was then a Visiting Professor with the University Carlos III, Madrid. He is currently a Research Fellow with the AUTOPIA Program, the Center for Automation and Robotics, Universidad Politécnica de Madrid-Consejo Superior de Investigaciones Científicas, Madrid. His research interests include nonlinear and optimal control and nonlinear estimation for intelligent transportation systems and robust control systems for humanoid and mobile robotic applications.

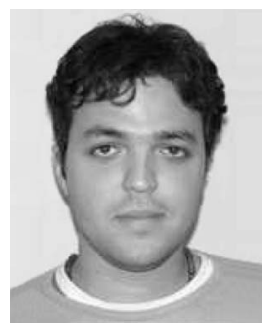

Joshué Pérez was born in Coro, Venezuela, in 1984. He received the B.E. degree in electronic engineering from Simón Bolívar University, Caracas, Venezuela, in 2007 and the M.E. degree in system engineering and automatic control from the University Complutense of Madrid, Madrid, Spain, in 2009. He is currently working toward the Ph.D. degree with the Center for Automation and Robotics, Universidad Politécnica de Madrid-Consejo Superior de Investigaciones Científicas, Madrid.

His research interests include fuzzy logic, modeling, control, and cooperative maneuvers among autonomous vehicles. 